\title{
Erratum: Construction and classification of point-group symmetry-protected topological phases in two-dimensional interacting fermionic systems [Phys. Rev. B 101, 100501(R) (2020)]
}

\author{
Jian-Hao Zhang, Qing-Rui Wang, Shuo Yang, Yang Qi, and Zheng-Cheng Gu $\odot$
}

(Received 8 February 2021; published 15 March 2021)

DOI: 10.1103/PhysRevB.103.099902

There are some minor errors in this paper. The main results remain correct, but the following corrections should be made:

(1) In the last row, the second column of Table I should be as follows:

$$
\begin{aligned}
\mathbb{Z}_{2}, & \text { if } m \in \text { odd, } \\
\mathbb{Z}_{2} \times \mathbb{Z}_{2}, & \text { if } m \in \text { even. }
\end{aligned}
$$

For odd $m$, the $\mathbb{Z}_{2}$ index is from the complex fermion decoration at the center of the dihedral group [for more details, see (5)]. Unlike the $D_{4}$ case with even $m$, the one-dimensional (1D) block-state decoration is actually not allowed for all odd $m$ 's. Here we demonstrate $D_{2}$ as a simplest example: Similar to the $D_{4}$ case, the Majorana chain decoration is not allowed. However, the 1D fermionic symmetry-protected topological (FSPT) state decoration leaves four dangling Majorana modes at the $D_{2}$ center with the following rotation and reflection symmetry properties:

$$
\boldsymbol{R}:\left(\eta_{1}, \eta_{1}^{\prime}, \eta_{2}, \eta_{2}^{\prime}\right) \mapsto\left(\eta_{2}, \eta_{2}^{\prime}, \eta_{1}, \eta_{1}^{\prime}\right) ; \quad \boldsymbol{M}:\left(\eta_{1}, \eta_{1}^{\prime}, \eta_{2}, \eta_{2}^{\prime}\right) \mapsto\left(\eta_{1},-\eta_{1}^{\prime}, \eta_{2},-\eta_{2}^{\prime}\right) .
$$

In order to gap them out, we add an interacting Hamiltonian $H_{U}=U \eta_{1} \eta_{1}^{\prime} \eta_{2} \eta_{2}^{\prime}(U>0)$. This Hamiltonian gives a gap but leaves a twofold ground-state degeneracy (GSD). In order to lift the GSD and obtain a gapped nondegenerate ground state, we should further consider some possible mass terms. All possible mass terms and their rotation properties are shown below

$$
\boldsymbol{R}:\left(\eta_{1} \eta_{2}, \eta_{1} \eta_{1}^{\prime}, \eta_{1} \eta_{2}^{\prime}, \eta_{2} \eta_{1}^{\prime}, \eta_{2} \eta_{2}^{\prime}, \eta_{1}^{\prime} \eta_{2}^{\prime}\right) \mapsto\left(-\eta_{1} \eta_{2}, \eta_{2} \eta_{2}^{\prime}, \eta_{2} \eta_{1}^{\prime}, \eta_{1} \eta_{2}^{\prime}, \eta_{1} \eta_{1}^{\prime},-\eta_{1}^{\prime} \eta_{2}^{\prime}\right)
$$

Thus, there are only two mass terms that are symmetric under $\boldsymbol{R}: \eta_{1} \eta_{1}^{\prime}+\eta_{2} \eta_{2}^{\prime}$ and $\eta_{1} \eta_{2}^{\prime}+\eta_{2} \eta_{1}^{\prime}$ and their linear combinations. Nevertheless, they are not symmetric under reflection,

$$
\boldsymbol{M}:\left(\eta_{1} \eta_{1}^{\prime}+\eta_{2} \eta_{2}^{\prime}, \eta_{1} \eta_{2}^{\prime}+\eta_{2} \eta_{1}^{\prime}\right) \mapsto\left[-\left(\eta_{1} \eta_{1}^{\prime}+\eta_{2} \eta_{2}^{\prime}\right),-\left(\eta_{1} \eta_{2}^{\prime}+\eta_{2} \eta_{1}^{\prime}\right)\right] .
$$

Therefore, there is no symmetric mass term to lift the GSD, and such a decoration is not allowed.

(2) In the paragraph following Eq. (13), the sentence "This construction can also be applied here and the zero-dimensional (OD) block state with odd fermion parity will also be trivialized" should be replaced by "The OD block state with odd fermion parity cannot be trivialized because this construction is not compatible with the reflection symmetry." [For more details, see (5).]

(3) In the last row, the last column of Table II should be as follows [see (6) and (7) for more detailed explanations]:

$$
\begin{aligned}
\mathbb{Z}_{2}, & \text { if } m \in \text { odd, } \\
\mathbb{Z}_{2} \times \mathbb{Z}_{2}, & \text { if } m \in \text { even. }
\end{aligned}
$$

(4) In the paragraph following Table II, the sentence "Spinless and spin- $1 / 2$ fermion systems with $D_{2}$ and $D_{6}$ point-group symmetry can also be constructed in a similar way and the classification results are exactly the same as the $D_{4}$ case." should be replaced by "Spinless and spin- $1 / 2$ fermion systems with $D_{2}$ and $D_{6}$ point-group symmetries can also be constructed in a similar way, but the classification results of spinless fermion systems with $D_{2}$ and $D_{6}$ symmetries is not identical with the $D_{4}$ case due to the absence of the 1D FSPT decoration."

(5) In Sec. V of the Supplemental Material, this construction is actually not compatible with the $D_{4}$ symmetry for spinless fermion systems. There are two possible cases: The reflection axis crosses the atomic sites or entanglement pairs of Majorana fermions, see Fig. 1. For the first case, consider the site containing the Majorana fermions $\gamma_{1}$ and $\gamma_{8}$ (see the left panel of Fig. 1), then the two-dimensional local Hilbert space is spanned by $|0\rangle$, and $c^{\dagger}|0\rangle\left[c^{\dagger}=\left(\gamma_{1}+i \gamma_{8}\right) / 2\right]$ forms a projective representation of $\mathbb{Z}_{2}^{f} \times \mathbb{Z}_{2}$ (the second $\mathbb{Z}_{2}$ is from reflection symmetry acting internally): In this Hilbert space, we have $\gamma_{1}=\sigma^{x}, \gamma_{8}=\sigma^{y}, P_{f}=$ $\sigma^{z}$, and $\boldsymbol{M}=\sigma^{x}+\sigma^{y}$ ( $\sigma^{x, y, z}$ are Pauli matrices). This representation satisfies the spinless condition: $\boldsymbol{M}^{2}=1$, and it is easy to see that $\boldsymbol{M} P_{f}=-P_{f} \boldsymbol{M}$ : The fermion parity is not compatible with the reflection symmetry. Apparently, the second case is incomparable with reflection symmetry. 

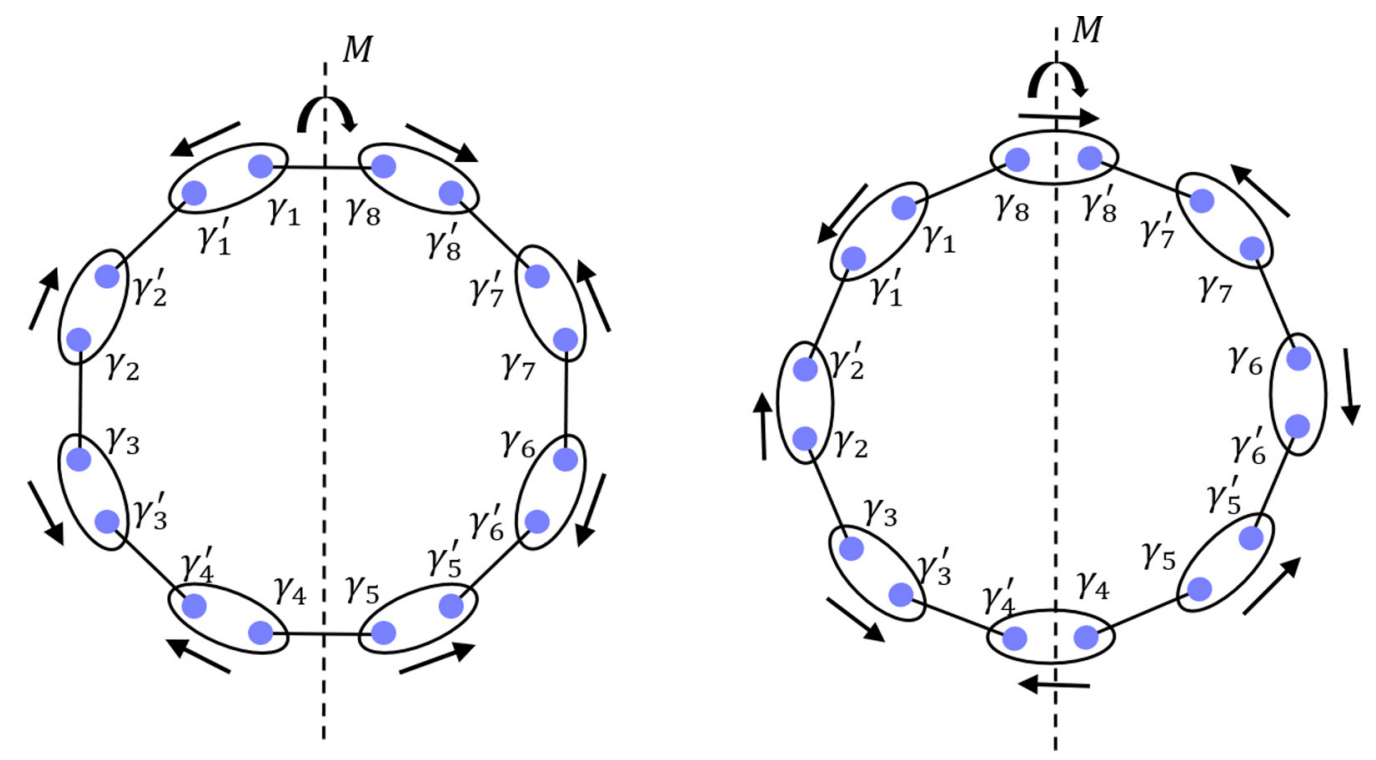

FIG. 1. Two possible arrangements of the Majorana chain with reflection symmetry. Left panel: the reflection axis crosses the atomic sites; right panel: the reflection axis crosses the entanglement pairs. Here each ellipse expresses an entanglement pair, each link represents an atomic site, and the dashed line represents the reflection axis.

(6) In Eq. (43) of the Supplemental Material, the correct three generators of $\mathcal{H}^{2}\left(D_{n}, \mathbb{Z}_{2}\right)$ should be as follows:

$$
\begin{aligned}
& n_{2}(a, b)=\left\lfloor\frac{\left[(-1)^{a_{y}+b_{y}} a_{x}\right]_{n}+\left[(-1)^{b_{y}} b_{x}\right]_{n}}{n}\right\rfloor+\left(1-\delta_{a_{x}}\right) b_{y}, \\
& n_{2}(a, b)=a_{x} b_{y}, \quad n_{2}(a, b)=a_{y} b_{y},
\end{aligned}
$$

where $\delta_{a_{x}}=1$ if $a_{x}=0$, otherwise, $\delta_{a_{x}}=0$. For spin- $1 / 2$ fermions, the correct $\omega_{2}$ should be as follows:

$$
\omega_{2}(a, b)=\left\lfloor\frac{\left[(-1)^{a_{y}+b_{y}} a_{x}\right]_{n}+\left[(-1)^{b_{y}} b_{x}\right]_{n}}{n}\right\rfloor+\left(1-\delta_{a_{x}}\right) b_{y}+a_{y} b_{y} .
$$

(7) In the second paragraph from the back of the Supplemental Material, the correct statement should be "for $n \equiv 0(\bmod 4)$, all $n_{2}$ 's are obstruction free; for $n \equiv 2(\bmod 4)$, one of those $n_{2}$ 's is obstructed. And the $n_{2}=\omega_{2}$ case should be trivialized. So the ultimate classifications of this case are $\mathbb{Z}_{2}^{2}$ for $n \equiv 0(\bmod 4)$ and $\mathbb{Z}_{2}$ for $n \equiv 2(\bmod 4)$." 